\title{
Deduction of Steady-State Cable Quench Limits for Various Electrical Insulation Schemes with Application to LHC and HL-LHC Magnets
}

\author{
Pier Paolo Granieri, Rob van Weelderen
}

\begin{abstract}
Undesired quenches of superconducting magnets can be a limiting factor for the operation of the LHC accelerator, both for its forthcoming exploitation at full energy as well as for its future upgrades. An accurate knowledge of the quench limit, the maximum amount of heat deposit the magnets can withstand, is required to be able to prevent beam induced quenches.

In this paper we provide an overview of the heat extraction through the multitude of cable insulation schemes used in particle accelerators in the past 20 years and foreseen for the coming years. Based on the relevant heat transfer measurements, we deduce steady-state cable quench limits both for the LHC $\mathrm{Nb}$-Ti magnets and for the future HL-LHC $\mathrm{Nb}_{3} \mathrm{Sn}$ ones. We provide them for different operating conditions and different locations within the coil.
\end{abstract}

Index Terms-Electrical insulation, heat transfer, superconducting high field magnets, LHC, LHC upgrade, $\mathrm{Nb}_{3} \mathrm{Sn}$, Nb-Ti, quench limit.

\section{INTRODUCTION}

$\mathrm{T}$ HE OPERATION of the Large Hadron Collider (LHC) particle accelerator at CERN [1] requires prevention of beam induced quenches, i.e. irreversible resistive transitions of the superconducting magnets caused by beam losses' heat deposit. Such quenches reduce the beam time hence the integrated luminosity. They represent as well a small risk of magnet failure, thus being detrimental for the accelerator exploitation.

In order to avoid beam induced quenches, it is required on the one hand to determine the heat deposit over the coil. This is obtained by combining calculations performed using the particle physics MonteCarlo simulation package FLUKA [2] with the signal coming from the Beam Loss Monitors (BLMs). The BLMs are detectors installed outside the magnet cryostats, measuring the flux of secondary particles (hadronic shower) produced during the impact of protons with magnet components [3].

On the other hand it is necessary to thoroughly determine the maximum heat deposit the magnet can withstand without experiencing a quench, hereafter referred to as quench limit.

An accurate knowledge of the quench limits allows

Manuscript received July 12, 2013. This research was supported by EU FP7 HiLumi LHC http://hilumilhc.web.cern.ch.

P.P. Granieri and R. van Weelderen are with CERN, Technology Department, Cryogenics Group, Geneva, Switzerland (phone: +41227676277 ; fax: +41227669967; e-mail: Pier.Paolo.Granieri@cern.ch). determining safe accelerator parameters at the beginning of the run, thus being essential for an efficient operation of the machine. This is especially important for future exploitation of the LHC, after the first Long Shutdown (LS1), when it will run at full beam energy $(6.5-7 \mathrm{TeV})$ instead of $4 \mathrm{TeV}$ so far. The operating conditions will be closer to the magnets' critical surface, hence the temperature margin will be reduced.

Comprehension of quench limits is also crucial to design future superconducting magnets, as those for the High Luminosity (HL) and High Energy (HE) LHC upgrades [4].

The mechanisms determining magnet stability strongly depend on the beam loss time scale, which spans several orders of magnitude from microseconds until hours [5]. For short duration beam losses, the quench limits are usually expressed in $\mathrm{mJ} / \mathrm{cm}^{3}$. The most significant mechanism is in this case the heat transfer from strands to the helium located inside the cable, i.e. filling the interstices among the strands. According to numerical computations of the LHC cables' stability for transient heat deposits ([6], updated in 2013 [7]), the heat transfer towards the external bath is negligible for heating durations shorter than $0.1 \mathrm{~s}$, whereas it becomes dominant for heating durations longer than $5 \mathrm{~s}$.

This paper deals with the determination of such steady-state cable quench limits, which are expressed in $\mathrm{mW} / \mathrm{cm}^{3}$. We deduce them from thermal measurements of a stack of cables that represents the winding pack of an accelerator magnet. These values can be directly used to set beam abort thresholds in the BLMs in case of long duration beam losses, and constitute an input (the boundary conditions) for calculating quench limits in case of short duration beam losses.

In section II we review the experimental techniques used to measure heat transfer in superconducting coils. The results referring to the dominant past and future cable electrical insulations are reported in section III. In section IV we discuss the parameters affecting steady-state quench limits and describe the procedure to compute them. This procedure is applied to the case of LHC and HL-LHC magnets in section $\mathrm{V}$, allowing identifying the most critical cables.

\section{EXPERIMENTAL TECHNIQUE}

The most widespread method to thermally characterize superconducting coils is the so-called stack method. It allows measuring the heat transfer through the cable's electrical insulation, which usual represents the most severe barrier for heat extraction from the magnet [8]. 
The techniques used in the last 20 years featured differences in terms of experimental set-up and thermometry. The stack method was developed at CEA during the R\&D phase of the LHC main magnets [9] [10] [11]. Several insulating materials and technologies were compared using solid conductors, instead of real cables, which were heated by Joule effect to reproduce the beam loss heat deposit. Although the conductor surface was machined to reproduce the external cable surface, helium could not penetrate inside the conductor; therefore the cable cross-section below the $\lambda$ temperature $T_{\lambda}$ was not isothermal. The setup developed at KEK to qualify the LHC MQXA interaction region quadrupoles made use of resistive cables with the same geometry of the superconducting ones, thus allowing superfluid helium (He II) to fill the interstices among strands [12] [13]. Heat transfer studies carried out at CERN were performed in a first stage on a segment of a superconducting LHC dipole coil [14]. Heat in the sample was generated through the inter-strands contact resistance. The instrumentation, using small in-house built thermocouples, was conceived to be as little invasive as possible. However, since the temperature sensors were not placed on the hottest strands where the current was injected, not all details of the heat transfer curves could be caught [15].

The measurements considered in this paper were carried out at CERN in a second stage and combined features of the above mentioned techniques. They used resistive cables and a thermometry based on small thermocouples. The technique and the results are detailed in [16] [17] [18]. The cables were insulated and alternately stacked to compensate for the cable keystone, thus forming a rectangular stack. In case of polyimide insulations, typical of $\mathrm{Nb}-\mathrm{Ti}$ coils, the stack underwent the magnet curing cycle (although at a higher pressure, which is a conservative choice). It was later installed in a sample-holder allowing application of compressive pressure on the cables' large faces, ranging between 5 and $100 \mathrm{MPa}$, corresponding to the azimuthal pressure in the coil. The actual pressure acting on the sample at cryogenic temperature was assessed. In case of impregnated insulations, typical of $\mathrm{Nb}_{3} \mathrm{Sn}$ coils, a small pressure of about $6 \mathrm{MPa}$ was applied during the impregnation, whereas no pressure was applied during test. Au-Fe vs. Chromel thermocouples were inserted inside the cable strands, at different locations (cable center and cable edge) as shown in Fig. 1. Both cables' small faces were in direct contact with the He II bath at $1.9 \mathrm{~K}$. Different heating configurations allowed to simulate various beam loss scenarios.

It is worth mentioning the other techniques that can be employed to determine quench limits on full scale magnets:

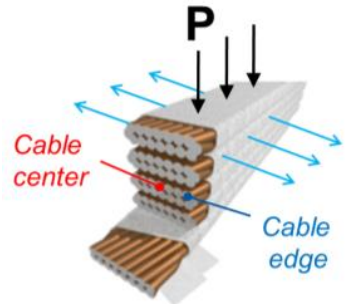

Fig. 1. Sketch of the stack used for the experimental studies [19]. quench induced by high current ramp rate [14] or by local heaters representing beam losses [20], as well as quench test with the beam [21].

\section{Heat Transfer through CABLE EleCtrical INSULATION}

Fig. 2 reports the evolution of electrical insulations of accelerator superconducting cables, in terms of heat transfer towards the He II cooling bath. The conditions assumed are the benchmark ones when comparing stack tests, i.e. both cables' small faces in contact with the bath at $1.9 \mathrm{~K}$, an applied pressure of $50 \mathrm{MPa}$ for insulations of $\mathrm{Nb}$-Ti cables, the three central cables heated where the middle one was instrumented, and temperature measured in the cable center.

Insulations of $\mathrm{Nb}-\mathrm{Ti}$ cables have been thermally improved

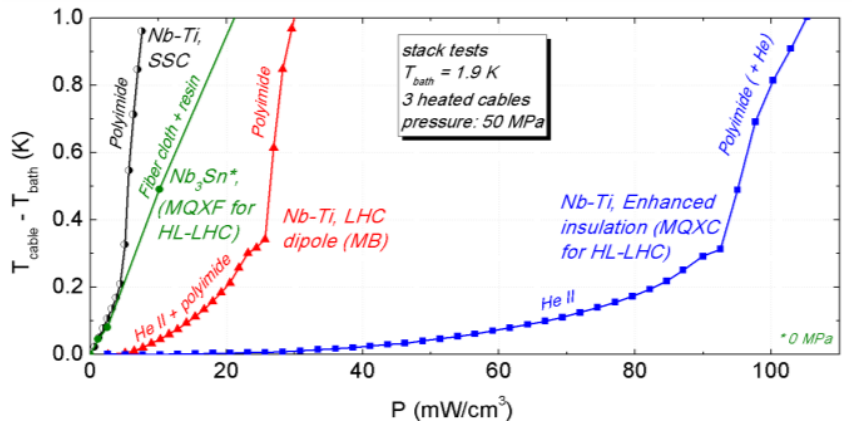

Fig. 2. Temperature difference between cable and bath until $1 \mathrm{~K}$ as a function of heat extracted in stack tests. Several electrical insulations of $\mathrm{Nb}-\mathrm{Ti}$ and $\mathrm{Nb}_{3} \mathrm{Sn}$ cables are considered (from [10], [16], [18] and [23]).

over the years, in particular concerning the micro-channels network created by the overlapping tapes. Such networks enabling a direct He II connection between strands and bath were gradually enhanced, thus providing an additional heat transfer mechanism besides solid conduction through polyimide. This improvement allowed extracting a larger amount of heat with the open insulation of the LHC dipole MB than with the almost sealed SSC insulation [10], at the same temperature level. A further improvement was obtained with the extremely open Enhanced Insulation of the $\mathrm{Nb}-\mathrm{Ti}$ HL-LHC interaction region (IR) quadrupole MQXC [16]. A good understanding of the underlying heat transfer mechanisms has been obtained [22] [23]. The situation is different for the insulation of $\mathrm{Nb}_{3} \mathrm{Sn}$ cables, as those for the $\mathrm{Nb}_{3}$ Sn HL-LHC IR quadrupole MQXF [24]. This insulation is

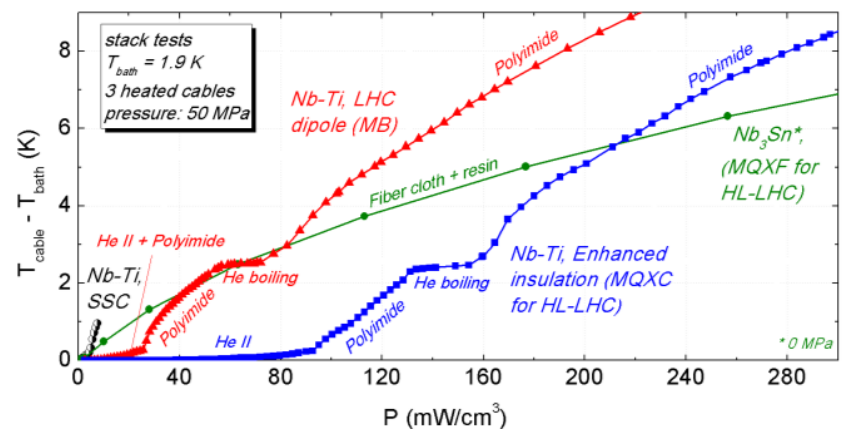

Fig. 3. Temperature difference between cable and bath until $9 \mathrm{~K}$ as a function of heat extracted in stack tests. Several electrical insulations of $\mathrm{Nb}-\mathrm{Ti}$ and $\mathrm{Nb}_{3} \mathrm{Sn}$ cables are considered (from [10], [18] and [23]). 
resin impregnated fiberglass not allowing helium to reach the strands [18]. Heat extraction relying in this case only on solid conduction through the insulation materials is worse than that through the LHC standard insulation, until a cable absolute temperature of $4 \mathrm{~K}$.

The majority of the quoted thermal studies focused on cable temperatures below $T_{\lambda}$ (including studies of the thermal properties of $\mathrm{Nb}_{3} \mathrm{Sn}$ cable's insulation [25] [26]). This may represent a design limit for $\mathrm{Nb}-\mathrm{Ti}$ coils, since the microchannels saturation above $T_{\lambda}$ results in the abrupt temperature rise shown in Fig. 2. However higher temperatures are of interest when investigating quench phenomena, as described in next section. In this case the heat transfer picture can drastically change, as shown in Fig. 3. At cable absolute temperatures above $8 \mathrm{~K}$ the insulation of $\mathrm{Nb}_{3} \mathrm{Sn}$ cables can extract more heat than the thermally Enhanced Insulation of $\mathrm{Nb}$-Ti cables. Though without micro-channels, it profits from higher thermal conductivity than polyimide.

\section{DEDUCTION OF Quench Limits}

Exceeding the superconductor current sharing temperature $T_{c s}$ following a transient heat pulse does not necessarily lead to a quench. It does in case of a steady-state heat deposit, if the heat generated by Joule heating is larger than the sum of heat removal through conduction along the cable and across the winding, and heat exchange with the coolant. Beam losses are typically spread several meters along the magnet axis and over several cables in the coil's cross-section [27]. As a consequence heat conduction is relevant only at the boundary of the affected volume and can be ignored for the majority of cables. For those cables it is appropriate to assume the heat extracted at $T_{c s}$ as the quench limit. $T_{c s}$ is about $4 \mathrm{~K}$ for $\mathrm{Nb}-\mathrm{Ti}$ and about $7 \mathrm{~K}$ for $\mathrm{Nb}_{3} \mathrm{Sn}$ in a $1.9 \mathrm{~K}$ bath [28], which is why it is of interest to extend the heat transfer curves well above $T_{\lambda}$.

It is worth noticing that the cable cross-section is uniformly heated in the stack test. This constitutes a difference with respect to the beam losses' radial profile featuring a maximum in the innermost strands [27]. This means that the deduced quench limits refer to an average heat deposit over the cable.

The cable quench limits depend on the following factors:

a. Heat extraction, which in turn depends on the:

i. cable cooling within the magnet;

ii. mechanical pressure, if $\mathrm{Nb}-\mathrm{Ti}$ coil;

iii. stack heating configuration.

b. Operating conditions, i.e.:

iv. transport current;

v. magnetic field, thus cable/strand considered.

The following procedure determines the quench limits reported in section V:

1. Experimentally correlate heat extraction and strands temperature. The heating configuration typically corresponds to heating all cables in the stack (around 5\% temperature difference w.r.t. the actual azimuthal beam losses' profile). In case of He II porous insulations for $\mathrm{Nb}$ - $\mathrm{Ti}$ coils, the heat extraction is influenced by the mechanical pressure on the cables' large faces. Therefore measurements are carried out at different pressures. The
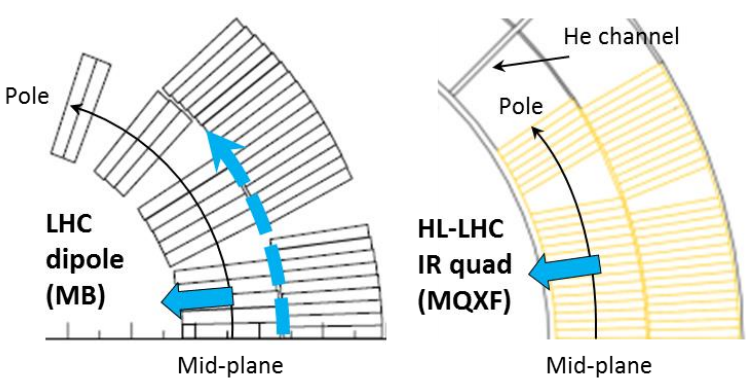

Fig. 4. One fourth of the coil of the LHC dipole MB (left) and of the HL-LHC insertion quadrupoles MQXF (right). Not to scale.

temperature is measured at different positions of the cable, in particular at the center and on the edge;

2. Scale the heat extraction to the coil geometry. As depicted in Fig. 4, only the innermost cables' small face is in direct contact with the He bath in the magnet annular space. The outermost small face can be, depending on magnet design, in contact with He II. This is the case for MB and MQXC $\mathrm{Nb}$-Ti coils, but not for the MQXF $\mathrm{Nb}_{3} \mathrm{Sn}$ coil. Therefore we scaled the heat extraction from the stack to the coil geometry for all temperature levels in case of MQXF, and only above $T_{\lambda}$ in case of MB and MQXC. The scaled curves are reported in Fig. 5.

3. Compute $T_{c s}$ using the known parameterizations [29] [30], as a function of transport current $I_{o p}$, hence beam energy, and magnetic field $B$ [31]. The latter depends on the cable location within the coil cross-section, as well as on the strand location within the cable cross-section (cable center or cable edge);

4. Compute the heat extracted at $T_{c s}\left(I_{o p}, B\right)$, as shown in Fig. 5 for the MB curve. For Nb-Ti coils, such computation is done at the pressure corresponding to the cable location within the coil cross-section, by interpolating on the measured heat transfer curves. From the mid-plane to the pole region, the pressure was assumed to vary between 50 and $5 \mathrm{MPa}$ for $\mathrm{MB}$ [32] and between 120 and $25 \mathrm{MPa}$ for MQXC [33]. For MQXF no pressure dependence was taken into account.

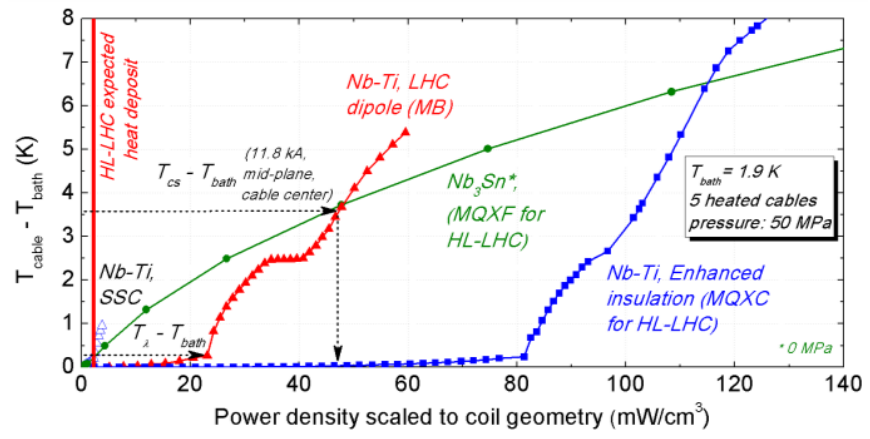

Fig. 5. Temperature difference between cable and bath as a function of extracted heat scaled to coil geometry. Several electrical insulations of $\mathrm{Nb}-\mathrm{Ti}$ and $\mathrm{Nb}_{3} \mathrm{Sn}$ cables are considered.

\section{QUENCH LIMITS}

In this section we present quench limit calculations for the coil of the LHC dipole (MB) and of the HL-LHC IR quadrupoles MQXC and MQXF. The bath temperature is 


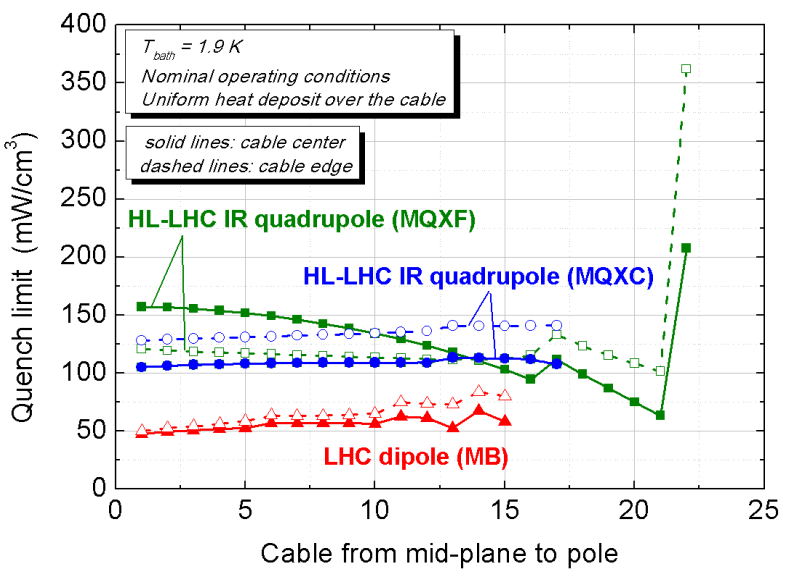

Fig. 6. Quench limit along the coil azimuthal direction for the inner coil of $\mathrm{MB}, \mathrm{MQXC}$ and MQXF.

$1.9 \mathrm{~K}$, held constant during heat removal. Fig. 6 reports the quench limit for each cable of the coil inner layer, along the azimuthal path going from the mid-plane to the pole. We considered the nominal current, i.e. $11.85 \mathrm{kA}$ for MB, $12.8 \mathrm{kA}$ for MQXC and 17.3 kA for MQXF. The quench limit is provided for the cable center and edge, representing the heat extracted until that part of the cable reaches $T_{c s}$. Whether the center is more or less stable than the edge depends on the interplay of magnetic field, typically higher at the edge, and temperature that is smaller at the edge for the same heat extraction. Both MQXC and MQXF coils feature a higher stability than MB.

In case of MQXF, as one would expect, the quench limits decrease as the magnetic field increases. Such a trend is more evident for the cable center curve, passing from above to below the cable edge curve when moving from mid-plane to pole, because of the higher field variation. Despite the higher magnetic field, the cable closer to the pole has a higher quench limit than the rest of the coil. The reason is its contact to the $\mathrm{Ti}$ pole piece, which is cooled by He II channels to enhance heat extraction from the magnet. The heat extracted from the cable closer to the pole is determined by scaling (50\%) the results obtained with the one heated cable configuration, whereas for all the other cables we considered the configuration with five heated cables, applying the scaling discussed in section IV. As a consequence, for cables 18 through 22 the increased heat

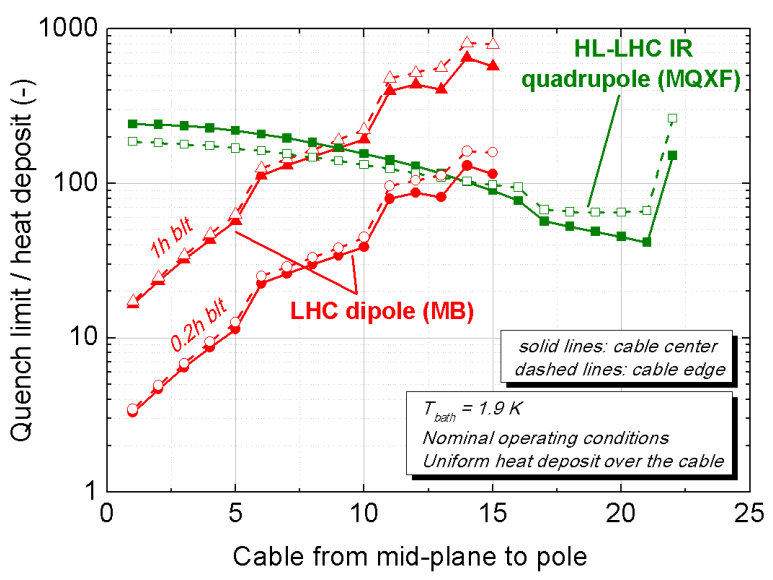

Fig. 7. Quench limit / heat deposit along the coil azimuthal direction for the inner coil of MB and MQXF. transfer between cables towards the Ti piece is not considered. This explains the discontinuity between the last two cables. The actual quench limit curve is expected to feature such behavior, though smoothen out when considering all the heat transfer mechanisms.

It is worth noticing that the provided quench limits refer to the cables. In other words, they correspond to the MQXF magnet quench limits as long as the channels through the $\mathrm{Ti}$ piece do not saturate [34], which might take few seconds.

As for the Nb-Ti coils MB and MQXC, the quench limits increase when moving towards the pole. This behavior, which might seem incoherent at first glance, is due to the dependence of the heat extraction on the mechanical pressure. When the magnet is powered, the Lorentz forces exert a higher pressure on the mid-plane cable than on the pole cable. This results in an increasing heat extraction when moving towards the pole.

Despite the fact that below $T_{\lambda}$ the MQXC insulation allows to extract four times more heat than the MB one (Fig. 2), the MQXC stability is only twice as large as MB. This is due to the similar slope of the relevant heat transfer curves above $T_{\lambda}$ (Fig. 3 and 4), where $T_{c s}$ is.

For all the magnets analyzed, the smallest quench limit is located in the cable center. Although taken as reference in the past [8] [40], this value is nevertheless influenced by the material properties of the cable used in the test. The quench limit referring to the cable edge is expected to be closer to reality, because less dependent on such properties and because the beam losses are mainly concentrated in the cable edge.

Another feature is the change of slope corresponding to the magnetic field discontinuities at the $\mathrm{Cu}$ wedges locations. This is particularly evident for the four MB cables near the pole, since the value of $T_{c s}$ is close to the plateau value associated to He boiling phenomena (Fig. 5). As a consequence, a small variation of $T_{c s}$ between the two sides of the $\mathrm{Cu}$ wedge may result in a significant variation of heat extraction.

In order to determine the most critical cables, we compare in Fig. 7 the quench limit in the cable center to the expected heat deposit (except for MQXC, where such data are not available). We consider the longitudinal peak loss rate that, for coherence, is also averaged over the cable cross-section. For $\mathrm{MB}$, the heat deposit [35] [36] refers to a beam energy of 6.5 $\mathrm{TeV}$, a loss rate of $4.5 \cdot 10^{11}$ protons $\cdot \mathrm{s}^{-1}$ and a relaxed

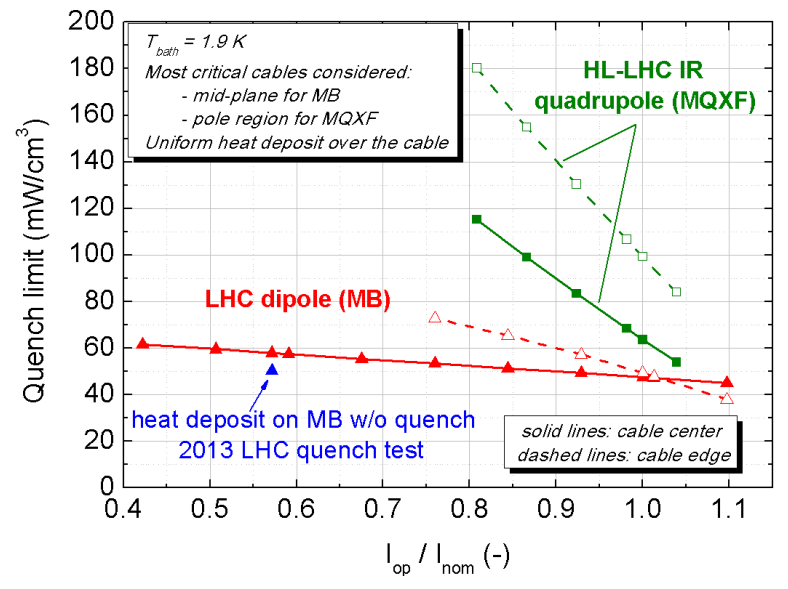

Fig. 8. Quench limit vs. operating / nominal current, for the most critical inner layer cable of MB and MQXF. The heat deposited during the 2013 LHC collimation quench test, which did not lead to a quench, is also reported. 
(pessimistic) scenario on the collimator settings (TCP7 at 6.7 $\sigma$, TCS7 at $9.9 \sigma$ [37]). We consider two possible beam lifetimes (blt): either 1 hour or the design specification of 0.2 hour. For MQXF, the heat deposit refers to a beam energy of 6.5 TeV and a luminosity of $5 \cdot 10^{34} \mathrm{~cm}^{-2} \mathrm{~s}^{-1}$ [38]. For both magnets the cables featuring the smallest quench limit are unluckily located in the most heated regions, i.e. the mid-plane area for MB (heat deposit of $3.4 \mathrm{~mW} / \mathrm{cm}^{3}$ or $16.8 \mathrm{~mW} / \mathrm{cm}^{3}$ for 1 hour and 0.2 hour blt, respectively) and the pole area for MQXF $\left(2.1 \mathrm{~mW} / \mathrm{cm}^{3}\right)$. The reason lies in the magnetic field for $\mathrm{MB}$ and in the shielding inside the magnet aperture for MQXF, which is thicker at the mid-plane. As a consequence the variation of the quench-limit / heat-deposit ratio along the azimuthal direction is higher than that of the quench limit. However the mentioned ratio is high enough, except in the pessimistic case of 0.2 hour blt, where in the worst-case (center of the mid-plane cable) it is only slightly larger than 3.

Fig. 8 reports the quench limits calculated in the above quoted most critical cable, as a function of the ratio between operating and nominal current. The quench limits decrease as the current increases. For MQXF the cable center curve is always the most critical one, whereas for MB that is the case until the nominal current.

We also compare our results to the LHC collimation quench test at $4 \mathrm{TeV}$ that took place in 2013 [39]. The loss rate was $1.63 \cdot 10^{12}$ protons $\cdot \mathrm{s}^{-1}$, obtained with collimation settings (TCP7 at $6.1 \sigma$, TCS7 at $10.1 \sigma$ [37]) that were more open than during standard operation, thus creating higher losses in the magnets. The heat deposit, averaged over the inner layer cable, was calculated to be $50 \mathrm{~mW} / \mathrm{cm}^{3}$ [35] [36]. This heat deposit did not lead to a quench, therefore in agreement with our quench limits. However, it must be noticed that the power deposited in the coil during the quench test was near steadystate. Numerical studies suggest that the quench limit in such conditions should be higher than the steady-state value [6] [7].

TABLE I

CABLE QUENCH LIMITS OF LHC AND HL-LHC MAGNETS

\begin{tabular}{cccc}
\hline \hline Magnet & \multirow{2}{*}{$\mathrm{SC}$} & $\begin{array}{c}\text { Operating } \\
\text { current }(\mathrm{kA})\end{array}$ & $\begin{array}{c}\text { Quench limit in the cable } \\
\text { center }- \text { edge }\left(\mathrm{mW} / \mathrm{cm}^{3}\right)\end{array}$ \\
\hline \multirow{2}{*}{$\mathrm{MB}$} & \multirow{2}{*}{$\mathrm{Nb}-\mathrm{Ti}$} & $\begin{array}{c}6.8(4 \mathrm{TeV}) \\
11(6.5 \mathrm{TeV})\end{array}$ & $58-80$ \\
& & $11.8(7 \mathrm{TeV})$ & $47-57$ \\
MQXF & $\mathrm{Nb}_{3} \mathrm{Sn}$ & 17.3 & $63-99$
\end{tabular}

The values provided refer to the most critical cable determined above for each magnet. The bath temperature is $1.9 \mathrm{~K}$.

Table I summarizes the quench limit values for MB and MQXF in the most significant current and future operating conditions, obtained with the assumptions reported in the paper. The two values refer to the cable center and edge. Past estimations of the MB quench limits were smaller, ranging from $5 \mathrm{~mW} / \mathrm{cm}^{3}$ [40] to $12 \mathrm{~mW} / \mathrm{cm}^{3}$ [41] at $7 \mathrm{TeV}$ operation, in the same conditions of uniform heat deposit in the cable cross-section. The reason is that they were neglecting He II and $\mathrm{He}$ boiling heat transfer mechanisms, which allow extracting more heat from the cable than the only solid conduction through the cable insulation.

\section{FURTHER INVESTIGATIONS}

In order to improve the accuracy of the quench limit computation based on the presented method, more efforts are required both from the experimental and modeling standpoint.

As far as the measurement technique is concerned, the actual coil configuration must be addressed to obtain a deeper insight of the He II heat transport mechanisms occurring in the inter-layers region. Different cooling conditions must be studied, since during operation the He bath might be heated above the nominal temperature of $1.9 \mathrm{~K}$. Transient operating conditions must also be investigated. They are of interest for the machine operation, as for instance in case of the mentioned quench test.

As for the numerical modeling of heat transfer in the coil, this must be extended above the $T_{\lambda}$ region [17], thus allowing simulating the actual heat deposit pattern that cannot be experimentally reproduced.

The study must be extended to the whole magnet, since there might be other regions saturating before the coil inner layer cables considered in this paper, e.g. the coil outer layer cables, the He volume in the annular space, the channels through the Ti piece in MQXF, etc.

Quench limits calculations will be performed also for other LHC current and future magnets.

\section{CONCLUSION}

We demonstrated a general method to determine steadystate quench limits of superconducting magnets, by measuring heat transfer on cable stacks while taking into account the cable cooling within the magnet.

The method was successfully applied to the LHC main dipole magnets and to magnets foreseen for the High Luminosity LHC upgrade. It allowed identifying the most critical regions in the coil cross-section, i.e. the mid-plane area for the Nb-Ti LHC dipoles and in the pole area for the future $\mathrm{Nb}_{3}$ Sn HL-LHC interaction regions quadrupoles. In nominal operating conditions the cable quench limits are several times higher than the expected heat deposit. However, in the conservative assumption of 0.2 hour beam lifetime the most pessimistic quench limit for the LHC main dipoles is only three times larger than the expected heat deposit. We also compared our results to the LHC collimation quench test performed in 2013 at a beam energy of $4 \mathrm{TeV}$, obtaining a good agreement.

\section{ACKNOWLEDGMENT}

The HiLumi LHC Design Study is included in the High Luminosity LHC project and is partly funded by the European Commission within the Framework Programme 7 Capacities Specific Programme, Grant Agreement 284404.

We are indebted to D. Richter who started the stack thermal measurement campaign at CERN, and grateful to L. Bottura and L. Rossi for the useful comments. We thank F. Cerutti, L.S. Esposito and E. Skordis for providing heat deposit data, as well as M. Sapinski, S. Redaelli and R. Bruce for the discussions on the quench test. 


\section{REFERENCES}

[1] O. Bruning et al., LHC design report vol. 1. CERN, Geneva, Switzerland, 2004.

[2] G. Battistoni et al., "The FLUKA code: description and benchmarking", Proceedings of the Hadronic Shower Simulation Workshop, Fermilab, AIP Conf. Proc. 896, pp. 31-49, 2007.

[3] B. Dehning et al., Design of the beam loss monitoring system for the LHC Ring, LHC Project report 781, CERN, Switzerland, 2004.

[4] L. Rossi, L. Bottura, G. de Rijk, E. Todesco, "Advanced accelerator magnets for upgrading the LHC", IEEE Trans. Appl. Supercond., vol. 22, no. 3, 4002008, 2012.

[5] A. Verweij, "Quenches after LS1", Proceedings of Chamonix 2012 workshop on LHC performance, pp. 299-302, 2012.

[6] P.P. Granieri et al., "Stability analysis of the LHC cables for transient heat depositions", IEEE Trans. Appl. Supercond., vol. 18, no. 2, pp. 1257-1262, 2008.

[7] P.P. Granieri et al., "Stability maps of the LHC superconducting magnets", IEEE Trans. Appl. Supercond., to be submitted for publication.

[8] L. Burnod, D. Leroy, B. Szeless, B. Baudouy, C. Meuris, "Thermal modeling of the LHC dipoles functioning in superfluid helium", Proceedings 4th European particle accelerator conference EPAC '94, London, England, pp. 2295-2297, 1994.

[9] C. Meuris, "Heat transport in insulation of cables cooled by superfluid helium", Cryogenics, vol. 31, pp. 624-628, 1991.

[10] C. Meuris, B. Baudouy, D. Leroy, B. Szeless, "Heat transfer in electrical insulation of LHC cables cooled with superfluid helium", Cryogenics, vol. 39, pp. 921-931, 1999.

[11] B. Baudouy, Etude des transferts de chaleur dans les isolations électriques de câbles supraconducteurs d'aimant d'accélérateur refroidi par hélium superfluide, Ph.D. dissertation, Université Pierre et Marie Curie and CEA-Saclay, France, 1996.

[12] N. Kimura et al., "Heat transfer from insulated Rutherford-type cables immersed in pressurized He II", Adv. Cryogenic Eng., vol. 43, pp. 14331440, 1998.

[13] N. Kimura, A. Yamamoto, T. Shintomi, A. Terashima, V. Kovachev, M. Murakami, "Heat transfer characteristics of Rutherford-type superconducting cables in pressurized He II", IEEE Trans. Appl. Supercond., vol. 9, no. 2, pp. 1097-1100, 1999.

[14] D. Richter et al., "Evaluation of the transfer of heat from the coil of the LHC dipole magnet to helium II", IEEE Trans. Appl. Supercond., vol. 17, no. 2, pp. 1263-1268, 2007.

[15] D. Richter, unpublished measurements, 2011.

[16] P.P. Granieri, P. Fessia, D. Richter, D. Tommasini, "Heat transfer in an enhanced cable insulation scheme for the superconducting magnets of the LHC luminosity upgrade", IEEE Trans. Appl. Supercond., vol. 20, no. 3, pp. 168-171, 2010.

[17] P.P. Granieri, Heat transfer between the superconducting cables of the LHC accelerator magnets and the superfluid helium bath, Ph.D. dissertation, Swiss Federal Institute of Lausanne (EPFL) and CERN, Switzerland, 2012.

[18] P.P. Granieri, L. Hincapié, R. van Weelderen, "Heat transfer through electrical insulation of $\mathrm{Nb}_{3}$ Sn cables”, IEEE Trans. Appl. Supercond. 23 submitted for publication.

[19] S. Pietrowicz, A. Four, B. Baudouy, N. Kimura, A. Yamamoto, "Heat dissipation in accelerator superconducting cables with ceramic insulation in normal and supercritical helium", Advances in Cryogenic Engineering, vol. 57A and 57B, no. 1434, pp. 1969-1975, 2012.

[20] G.A. Kirby et al., "LHC IR upgrade $\mathrm{Nb}-\mathrm{Ti}, 120 \mathrm{~mm}$ aperture model quadrupole test results at $1.8 \mathrm{~K}$ ", IEEE Trans. Appl. Supercond. 23 submitted for publication.

[21] M. Sapinski, "Quench limits", Proceedings of Chamonix 2012 workshop on LHC performance, pp. 121-127, 2012.

[22] B. Baudouy, M.X. François, F.P. Juster, C. Meuris, "He II heat transfer through superconducting cables electrical insulation", Cryogenics, vol. 40, pp. 127-136, 2000.

[23] P.P. Granieri, "Heat transfer through cable insulation of $\mathrm{Nb}-\mathrm{Ti}$ superconducting magnets operating in He II', Cryogenics, vol. 53, pp. 61-71, 2013.

[24] P. Ferracin et al., "Magnet design of the $150 \mathrm{~mm}$ aperture low- $\beta$ quadrupoles for the High Luminosity LHC", IEEE Trans. Appl. Supercond. 23 submitted for publication.
[25] B. Baudouy, J. Polinski, "Thermal conductivity and Kapitza resistance of epoxy resin fiberglass tape at superfluid helium temperature", Cryogenics, vol. 49, pp. 138-143, 2009.

[26] S. Pietrowicz, A. Four, S. Jones, S. Canfer, B. Baudouy, "Thermal conductivity and Kapitza resistance of cyanate ester epoxy mix and trifunctional epoxy electrical insulations at superfluid helium temperature", Cryogenics, vol. 52, pp. 100-104, 2012.

[27] F. Cerutti et al., Estimation of threshold for the signals of the BLMs around the LHC final focus triplet magnets, LHC Project report ATSNote-2012-014, CERN, Geneva, Switzerland, 2012.

[28] E. Todesco, P. Ferracin, "Limits to high field magnets for particle accelerators", IEEE Trans. Appl. Supercond., vol. 22, no. 3, 4003106, 2012.

[29] L. Bottura, "A practical fit for the critical surface of Nb-Ti", IEEE Trans. Appl. Supercond., vol. 10, pp. 1054-1057, 2000.

[30] L.T. Summers, et al., "A model for the prediction of $\mathrm{Nb}_{3} \mathrm{Sn}$ critical current as a function of field, temperature, strain and radiation damage", IEEE Trans. Magnetics, vol. 27, pp. 2041-2044, 1991.

[31] P. Hagen and S. Izquierdo Bermudez, private communication.

[32] P. Ferracin, Superconducting accelerator magnets. Unit 14: Construction methods and support structures. Episode II, US Particle Accelerator School (USPAS), 2007.

[33] G.A. Kirby, " Engineering design and manufacturing challenges for a wide-aperture, superconducting quadrupole magnet”, IEEE Trans. Appl. Supercond., vol. 22, no. 3, 4001804, 2012.

[34] J.M. Poncet, H. Allain, R. van Weelderen, "Cooling options for the LHC high luminosity upgrade final focusing magnets", Advances in Cryogenic Engineering, submitted for publication.

[35] E. Skordis, "Energy deposition simulations for quench tests", presented at the LHC collimation review 2013, CERN, Switzerland, 2013.

[36] LHC collimation review 2013, website: http://indico.cern.ch/conferenceDisplay.py?confId=251588

[37] R. Bruce, private communication.

[38] L.S. Esposito, F. Cerutti, E. Todesco, "FLUKA energy deposition studies for the HL-LHC", Proceedings of IPAC 2013, TUPFI021, Shanghai, China.

[39] S. Redaelli, R. Bruce, B. Salvachua, D. Wollmann, Quench margin at $4 \mathrm{TeV}, \mathrm{LHC}$ Project report 1264646, CERN, Switzerland, 2013.

[40] B.J. Jeanneret, D. Leroy, L.R. Oberli, T. Trenkler, Quench levels an transient beam losses in LHC magnets, LHC Project report 44, CERN, Switzerland, 1996.

[41] D. Bocian, B. Dehning, A. Siemko, "Quench limit model and measurement for steady state heat deposit in LHC magnets", IEEE Trans. Appl. Supercond., vol. 19, no. 3, pp. 2446-2449, 2009. 